\title{
ON THE EXTENDED HOLOMORPHIC CURVES ON $C^{*}$-ALGEBRAS
}

\author{
YINGLI HOU AND KUI JI
}

Abstract. For $\Omega \subseteq \mathbb{C}$ a connected open set, and $\mathscr{U}$ a unital $C^{*}$-algebra, let $\mathscr{P}(\mathscr{U})$ denote the sets of all projections in $\mathscr{U}$. If $P: \Omega \rightarrow \mathscr{P}(\mathscr{U})$ is a holomorphic $\mathscr{U}$-valued map, then $P$ is called an extended holomorphic curve on $\mathscr{P}(\mathscr{U})$. In this note, we focus on discussing the unitary classification problem of extended holomorphic curves. By considering the metric of related determinant jet bundles, we give a necessary and sufficient condition for some extended holomorphic curves on $C^{*}$-algebras to be unitary equivalent.

Mathematics subject classification (2010): Primary 47C15, 47B37; Secondary 47B48, 47L40.

Keywords and phrases: $C^{*}$-algebras, unitary equivalence, curvatures, extended holomorphic curves.

\section{REFERENCES}

[1] C. Apostol And M. Martin, A $C^{*}$-algebra approach to the Cowen-Douglas theory, Topics in modern operator theory (Timisoara/Herculane, 1980), pp. 45-51, Operator Theory: Adv. Appl., 2, Birkhäuser, Basel-Boston, Mass., 1981.

[2] M. J. Cowen And R. G. Douglas, Complex geometry and operator theory, Acta Math. 141 (1978), no. 3-4, 187-261.

[3] K. Ji, Similarity Classification and Properties of Some Extended Holomorphic Curves, Integr. Equ. Oper. Theory, 69 (2011), no. 1, 133-148.

[4] H. Kwon AND S. Treil, Similarity of operators and geometry of eigenvector bundles, Publ. Mat. (2009) Vol. 53, no. 2, 417-438.

[5] L. LI, On the classification of simple $C^{*}$-algebras: Inductive limits of matrix algebras over trees, Mem. Amer. Math. Soc., no. 605, vol. 127, (1997).

[6] M. Martin And N. Salinas, Flag Manifolds and the Cowen-Douglas theory, J. Operator theory. 38 (1997), no. 2, 329-365.

[7] M. MARTIN AND N. SAlinAS, The canonical complex structure of flag manifolds in a $C^{*}$-algebra, Nonselfadjoint operator algebras, operator theory, and related topics, 173-187, Oper. Theory Adv. Appl., 104, Birkhäuser, Basel, 1998.

[8] M. Martin, Hermitian geometry and involutive algebras, Math. Z. 188 (1985), no. 3, 359-382.

[9] M. Martin AND N. SAlinas, Differential geometry of generalized Grassmann manifolds in $C^{*}$ algebras, Operator theory and boundary eigenvalue problems (Vienna, 1993), 206-243, Oper. Theory Adv. Appl., 80, Birkhäuser, Basel, 1995.

[10] G. MisRA, Curvature and backward shift operators, Proc. Amer. Math. Soc. 91 (1984), no. 2, $105-$ 107.

[11] G. Misra, Curvature inequality and extremal properties of bundle shifts, J. Operator Theory 11 (1984), no. 1, 305-317.

[12] M. Rordam, F. Larsen And N. J. Laustsen, An introduction to K-Theory for $C^{*}$-algebras, London Mathematical Society Student Texts 49, Cambridge University Press, 2000.

[13] N. SAlinas, The Grassmann manifold of a $C^{*}$-algebra, and Hermitian holomorphic bundles, Special classes of linear operators and other topics (Bucharest, 1986), 267-289, Oper. Theory Adv. Appl., 28, Birkhäuser, Basel, 1988.

[14] M. UchiYama, Curvatures and similarity of operators with holomorphic eigenvectors, Tran. Amer. Math. Soc. 319 (1990), no. 1, 405-415. 